Research Paper

\title{
Early response and pathological complete remission in Breast Cancer with different molecular subtypes: a retrospective single center analysis
}

\author{
Jin Hong, Jiayi Wu, Ou Huang, Jianrong He, Li Zhu, Weiguo Chen, Yafen Li, Xiaosong Chen ${ }^{\varpi}$, Kunwei Shen ${ }^{\varpi \#}$ \\ Department of General Surgery, Comprehensive Breast Health Center, Ruijin Hospital, Shanghai Jiao Tong University School of Medicine, Shanghai, 200025, PR \\ China. \\ \#Co-corresponding authors with equal contributions to this work. \\ $\triangle$ Corresponding authors: Kunwei Shen, Telephone number: +86-21-64370045-602208; E-mail address: kwshen@medmail.com.cn, Complete address: \\ Comprehensive Breast Health Center, Ruijin Hospital, Shanghai Jiao Tong University School of Medicine, No.197 Ruijin Second Road, Huangpu District, \\ Shanghai, 200025, PR China; and, Xiaosong Chen, Telephone number: +86-21-64370045-602102; E-mail address: chenxiaosong0156@hotmail.com, Complete \\ address: Comprehensive Breast Health Center, Ruijin Hospital, Shanghai Jiao Tong University School of Medicine, No.197 Ruijin Second Road, Huangpu \\ District, Shanghai, 200025, PR China.
}

(c) The author(s). This is an open access article distributed under the terms of the Creative Commons Attribution License (https://creativecommons.org/licenses/by/4.0/). See http://ivyspring.com/terms for full terms and conditions.

Received: 2020.04.08; Accepted: 2020.09.06; Published: 2020.10.06

\begin{abstract}
Purpose: To evaluate the association of clinical early response and pathological complete remission (pCR) in breast cancer patients with different molecular subtypes.

Materials and methods: Breast cancer patients who received neoadjuvant treatment (NAT) with clinical early response assessment from October 2008 to October 2018 were retrospectively analyzed. Clinical early response was defined as tumor size decreasing $\geq 30 \%$ evaluated by ultrasound after two cycles of NAT. Chi-square test was used to compare the PCR rates between the responder and non-responder groups with different molecular subtypes. Multivariate logistic regression was used to identify independent factors associated with the $\mathrm{PCR}$.

Results: A total of 328 patients were included: 100 responders and 228 non-responders. The progesterone receptor (PR) expression was an independent factor associated with clinical early response $(\mathrm{OR}=2.39$, $95 \% \mathrm{Cl}=1.41-4.05, P=0.001)$. The $P C R$ rate of breast was $50.0 \%$ for responders and $18.0 \%$ for non-responders $(P<0.001)$. Regarding different molecular subtypes, responders had higher $P C R$ rates than non-responders for patients with HER2 overexpression $(O R=10.66,95 \% \mathrm{Cl}=2.18-52.15, P=0.001)$, triple negative $(\mathrm{OR}=3.29$, $95 \% \mathrm{Cl}=1.23-8.84, \quad P=0.016)$ and Luminal (HER2-) subtypes $(\mathrm{OR}=8.58,95 \% \mathrm{Cl}=3.05-24.10, \quad P<0.001)$ respectively. Moreover, $\mathrm{PCR}$ rate can be achieved as high as $88.2 \%$ in HER2 overexpression patients with early clinical response, which was significantly higher than patients without early response $(41.3 \%, P=0.001)$. Multivariate analysis showed that clinical early response was an independent factor associated with the $\mathrm{pCR}$ rate $(\mathrm{OR}=4.87,95 \% \mathrm{Cl}=2.72-8.72, P<0.001)$.

Conclusions: Early response was significantly associated with a higher PCR rate in breast cancer patients receiving NAT, especially for patients with HER2 overexpression subtype, which warrants further clinical evaluation.
\end{abstract}

Key words: Breast cancer; clinical early response; pathological complete response; neoadjuvant treatment

\section{Introduction}

Neoadjuvant treatment (NAT) is a common strategy to downstage locally advanced diseases and increase breast conservative surgery rate in breast cancer patients [1, 2], which was found to have similar disease outcome compared to patients receiving adjuvant chemotherapy $[3,4]$. Nevertheless, patients who had achieved pathological complete remission (pCR) after NAT had significantly better disease free survival (DFS) and overall survival (OS) than those without pCR [5, 6], which will accelerate drug efficacy evaluation and new drug approval.

As a result, achieving pCR becomes quite an 
important aim for better outcomes in NAT. The reported $\mathrm{pCR}$ rates varied with different phenotypes from $0 \%$ to $79 \%$ in previous studies [6]. The tumor biology is a critical factor associated with pCR after NAT, estrogen receptor (ER) and progesterone receptor (PR) negativity, human epidermal growth factor receptor-2 (HER2) positivity, high mitotic count and Ki67 score were correlated with pCR[6, 7]. A retrospective study from Memorial Sloan Kettering Cancer Center (MSKCC) found that patients with HER2 positive tumors had the highest $\mathrm{pCR}$ rate and the lowest $\mathrm{pCR}$ rate appeared in the hormone receptor (HR) positive HER2 negative subgroup [8].

Therapeutic response differs individually in breast cancer patients and clinical response is a method for early evaluation. Aside from molecular markers, clinical response to initial cycles of neoadjuvant chemotherapy appeared to be associated with the pCR rate [9]. Therefore, early identification of clinical response in neoadjuvant therapy may help us to select those who are responsive to initial regimens. For non-responders, it may provide an opportunity to get an alternative therapy. Few studies evaluated the predictive value of early response assessed by ultrasound for $\mathrm{pCR}$ in different breast cancer molecular subtypes and survival. In this study, we aimed to evaluate the association of clinical early response and $\mathrm{pCR}$ rate in breast cancer patients with different molecular subtypes as well as survival outcome.

\section{Materials and Methods}

\section{Patient population}

Six hundred and forty consecutive breast cancer patients who received preoperative treatment from October 2008 to October 2018 in Shanghai Jiao Tong University Breast Cancer Database (SJTU-BCDB) were analyzed retrospectively (Figure 1). Eligibility criteria were as follows: (1) invasive breast cancer; (2) received standard neoadjuvant chemotherapy and/or trastuzumab for $\geq 4$ cycles; (3) with clinical response assessment after two cycles of NAT; (4) with pathological evaluation after surgery. Patients with following criteria were excluded: (1) with distant metastasis; (2) received neoadjuvant endocrine therapy or non-standard chemotherapy (capecitabine or vinorelbine); (3) received NAT $<4$ cycles; (4) without clinical response assessment after two cycles of NAT.

All patients received neoadjuvant chemotherapy and patients with HER-2 positive could also receive trastuzumab. Anthracycline (A) and taxanes (T) based regimens included EC-T (epirubicin $90 \mathrm{mg} / \mathrm{m}^{2}$, cyclophosphamide $600 \mathrm{mg} / \mathrm{m}^{2}$ and followed by docetaxel $100 \mathrm{mg} / \mathrm{m}^{2}$ ) and TEC (docetaxel $75 \mathrm{mg} / \mathrm{m}^{2}$, epirubicin $75 \mathrm{mg} / \mathrm{m}^{2}$ and cyclophosphamide 500 $\mathrm{mg} / \mathrm{m}^{2}$ ) every 21 days. Anthracycline or taxanes based regimens included EC (epirubicin $90 \mathrm{mg} / \mathrm{m}^{2}$ and cyclophosphamide $600 \mathrm{mg} / \mathrm{m}^{2}$ ) or TC (docetaxel $75 \mathrm{mg} / \mathrm{m}^{2}$ and cyclophosphamide $600 \mathrm{mg} / \mathrm{m}^{2}$ ) every 21 days. Trastuzumab(H) was administrated $8 \mathrm{mg} / \mathrm{Kg}$ at first cycle and followed by $6 \mathrm{mg} / \mathrm{Kg}$ every 3 weeks or $4 \mathrm{mg} / \mathrm{Kg}$ at first cycle and followed by $2 \mathrm{mg} / \mathrm{Kg}$ every week. Targeted combined with chemotherapy regimens included $\mathrm{EC}-\mathrm{TH}, \mathrm{TCbH}$ (docetaxel 75 $\mathrm{mg} / \mathrm{m}^{2}$ and carboplatin AUC 6) every 21 days or $\mathrm{PCbH}$ (paclitaxel $80 \mathrm{mg} / \mathrm{m}^{2}$ and carboplatin AUC 2) every 7 days.

\section{Clinical and pathological evaluation}

Baseline patients' characteristics were obtained from the SJTU-BCDB, including age, menopausal status, clinical tumor stage, clinical nodal stage and neoadjuvant treatment cycles. Pathological characteristics were confirmed by core needle biopsy, including pathology type, tumor grade, ER, PR and C-erbB-2. ER and PR positivity were defined as more than $1 \%$ positive invasive tumor cells with nuclear staining. Tumors with immunohistochemical (IHC) HER-2 3+ and/or HER2 gene amplification confirmed by florescent in situ hybridization (FISH) were regarded as HER2 positive. Tumors were classified as four molecular subtypes: Luminal-HER2 negative (HR+HER-), Luminal-HER2 positive (HR+HER2+), HER2 overexpression (HR-HER2+) and triple negative breast cancer (TNBC, HR-HER2-).

Clinical response assessment was performed by three-dimensional (3D) ultrasound before NAT and after two cycles of NAT. The tumor response can be classified into four types: complete response (CR, disappearance of all target lesions), partial response (PR, at least a $30 \%$ decrease in the sum of diameters of target lesions), progressive disease (PD, at least a $20 \%$ increase in the sum of diameters of target lesions) and stable disease (SD, neither PD nor PR) according to the Response Evaluation Criteria in Solid Tumor (RECIST) version 1.1[10]. Patients with CR or PR after two cycles of NAT were defined as responders and patients with PD or SD were defined as nonresponders. Pathological response was evaluated after surgery. PCR in breast was defined as absence of invasive cancer in the breast, irrespective of DCIS or nodal involvement (ypT0/is) [11].

\section{Statistical analysis}

Categorical variables between two groups were compared using two-sided Pearson chi-square test. Multivariable logistic regression model was used to determine the independent predictive factor for $\mathrm{pCR}$ 
and variables with $p$ value $<0.05$ in the univariate analysis were included. DFS was defined as the interval from the day of surgery to the date of any event as follows: any local or regional recurrence, distant metastasis, newly diagnosed contralateral breast cancer, any secondary malignancy or death from any cause. OS was defined as the interval from the day of surgery to the date of death from any cause. DFS and OS were analyzed and compared using Kaplan-Meier method and the log-rank test. Statistical analyses were performed using SPSS (version 22.0) software (IBM Corporation, Armonk, NY, USA).

\section{Results}

\section{Patients' characteristics}

Between October 2008 and October 2018, 640 invasive breast cancer patients receiving preoperative treatment were collected from SJTU-BCDB. Among them, 328 patients who were treated with 4 or more cycles of NAT and assessed for clinical early response by $3 \mathrm{D}$ ultrasound were included in the subsequent analyses (Figure 1). Among those patients, 100 were responders and 228 were non-responders.

Patients' characteristics were summarized in Table 1 . The median age was 55 years old and $50 \%$ of the patients were premenopausal women. 263 patients had cT0 to CT2 disease and 65 patients had cT3 / 4 disease. 220 patients were diagnosed as cN0 or $\mathrm{cN} 1$ and 101 patients had $\mathrm{cN} 2 / 3$ disease. Among those patients, 138 patients were Luminal (HER2-) subtype, 53 patients were Luminal (HER2+) subtype, 63 patients were HER2 overexpression and 74 patients were triple negative breast cancer. 254 patients received anthracycline and taxanes $(\mathrm{A}+\mathrm{T})$ combined neoadjuvant chemotherapy, and 74 patients received anthracycline $(\mathrm{A})$ or taxanes $(\mathrm{T})$ based regimens. 86 of 116 HER-2 positive patients received trastuzumab. Among those patients, 76 patients received only 4 cycles of NAT and 252 patients received more than 4 cycles of treatment.

\section{Factors associated with clinical early response}

In the univariate analysis, the proportion of responders was significantly higher in ER negative tumors than ER positive tumors $(36.5 \%$ vs $26.2 \%, P=$ 0.045) and in PR negative tumors than PR positive tumors $(36.7 \%$ vs $19.7 \%, P=0.001)$ (Table 1$)$. The highest proportion of responders was $44.6 \%$ in the TNBC subgroup and the lowest was $24.6 \%$ in the Luminal (HER2-) group $(P=0.023)$. Multivariate analysis indicated PR was an independent factor for clinical early response $(\mathrm{OR}=2.39,95 \% \mathrm{CI}=1.41-4.05, P$ $=0.001$ ) (Table 2 ).
Table 1. Baseline Characteristics and Treatments

\begin{tabular}{|c|c|c|c|c|}
\hline Characteristics & $\begin{array}{l}\text { Total } \\
\mathrm{N}=328(\%)\end{array}$ & $\begin{array}{l}\text { Responders } \\
\mathrm{N}=100(\%)\end{array}$ & $\begin{array}{l}\text { Non-responders } \\
\mathrm{N}=228(\%)\end{array}$ & $P$-value \\
\hline Age, years & & & & 0.491 \\
\hline$<50$ & $157(47.9)$ & $45(28.7)$ & $112(71.3)$ & \\
\hline$\geq 50$ & $171(52.1)$ & $55(32.2)$ & $116(67.8)$ & \\
\hline Menopausal status & & & & 1.000 \\
\hline Premenopausal & $164(50)$ & $50(30.5)$ & $114(69.5)$ & \\
\hline Postmenopausal & $164(50)$ & $50(30.5)$ & $114(69.5)$ & \\
\hline Pathology & & & & 0.509 \\
\hline IDC & $272(82.9)$ & $85(31.2)$ & $187(68.8)$ & \\
\hline Others & $56(17.1)$ & $15(26.8)$ & $41(73.2)$ & \\
\hline Clinical T stage & & & & 0.147 \\
\hline T0-2 & $263(80.2)$ & $85(32.3)$ & $178(67.7)$ & \\
\hline T3-4 & 65 (19.8) & $15(23.1)$ & $50(76.9)$ & \\
\hline Clinical N stage & & & & 0.409 \\
\hline N0-1 & $220(68.5)$ & $64(29.1)$ & $156(70.9)$ & \\
\hline $\mathrm{N} 2-3$ & $101(31.5)$ & 34 (33.7) & $67(66.3)$ & \\
\hline Grade & & & & 0.674 \\
\hline I-II & $78(23.8)$ & $21(26.9)$ & 57 (73.1) & \\
\hline III & $144(43.9)$ & $44(30.6)$ & $100(69.4)$ & \\
\hline NA & $106(32.3)$ & $35(33.0)$ & $71(67.0)$ & \\
\hline ER & & & & 0.045 \\
\hline positive & $191(58.2)$ & $50(26.2)$ & $141(73.8)$ & \\
\hline negative & $137(41.8)$ & $50(36.5)$ & $87(63.5)$ & \\
\hline PR & & & & 0.001 \\
\hline positive & $122(37.2)$ & $24(19.7)$ & $98(80.3)$ & \\
\hline negative & $206(62.8)$ & $76(36.9)$ & $130(63.1)$ & \\
\hline HER-2 & & & & 0.553 \\
\hline positive & $116(35.4)$ & $33(28.4)$ & $83(71.6)$ & \\
\hline negative & $212(64.6)$ & $67(31.6)$ & $145(68.4)$ & \\
\hline Subtype & & & & 0.023 \\
\hline Luminal (HER2-) & $138(42.1)$ & $34(24.6)$ & $104(75.4)$ & \\
\hline Luminal (HER2+) & $53(16.2)$ & $16(30.2)$ & $37(69.8)$ & \\
\hline HER2 overexpression & $63(19.2)$ & $17(27.0)$ & $46(73.0)$ & \\
\hline TNBC & $74(22.6)$ & $33(44.6)$ & $41(55.4)$ & \\
\hline Regimens & & & & 0.065 \\
\hline $\mathrm{A}$ or $\mathrm{T}$ & 74 (22.6) & 29 (39.2) & $45(60.8)$ & \\
\hline $\mathrm{A}$ and $\mathrm{T}$ & $254(77.4)$ & $71(28.0)$ & $183(72.0)$ & \\
\hline Target therapy & & & & 0.250 \\
\hline Yes & $86(26.2)$ & $22(25.6)$ & $64(74.4)$ & \\
\hline No & $242(73.8)$ & $78(32.2)$ & $164(67.8)$ & \\
\hline Neoadjuvant cycles & & & & 0.814 \\
\hline 4 & $76(23.2)$ & $24(31.6)$ & $52(68.4)$ & \\
\hline$>4$ & $252(76.8)$ & $76(30.2)$ & $176(69.8)$ & \\
\hline
\end{tabular}

Table 2. Multivariate analysis for factors associated with clinical early response

\begin{tabular}{|c|c|c|c|}
\hline Characteristics & OR & $95 \% \mathrm{CI}$ & $P$-value \\
\hline \multicolumn{4}{|l|}{ ER status } \\
\hline positive & 1 & & \\
\hline negative & 1.37 & $0.66-2.86$ & 0.379 \\
\hline \multicolumn{4}{|l|}{ PR status } \\
\hline positive & 1 & & \\
\hline negative & 2.39 & $1.41-4.05$ & 0.001 \\
\hline Subtypes & & & 0.502 \\
\hline Luminal (HER2-) & 1 & & \\
\hline Luminal (HER2+) & 0.93 & $0.45-1.93$ & 0.841 \\
\hline HER2 overexpression & 1.59 & $0.71-3.53$ & 0.257 \\
\hline TNBC & 0.73 & $0.35-1.52$ & 0.397 \\
\hline
\end{tabular}




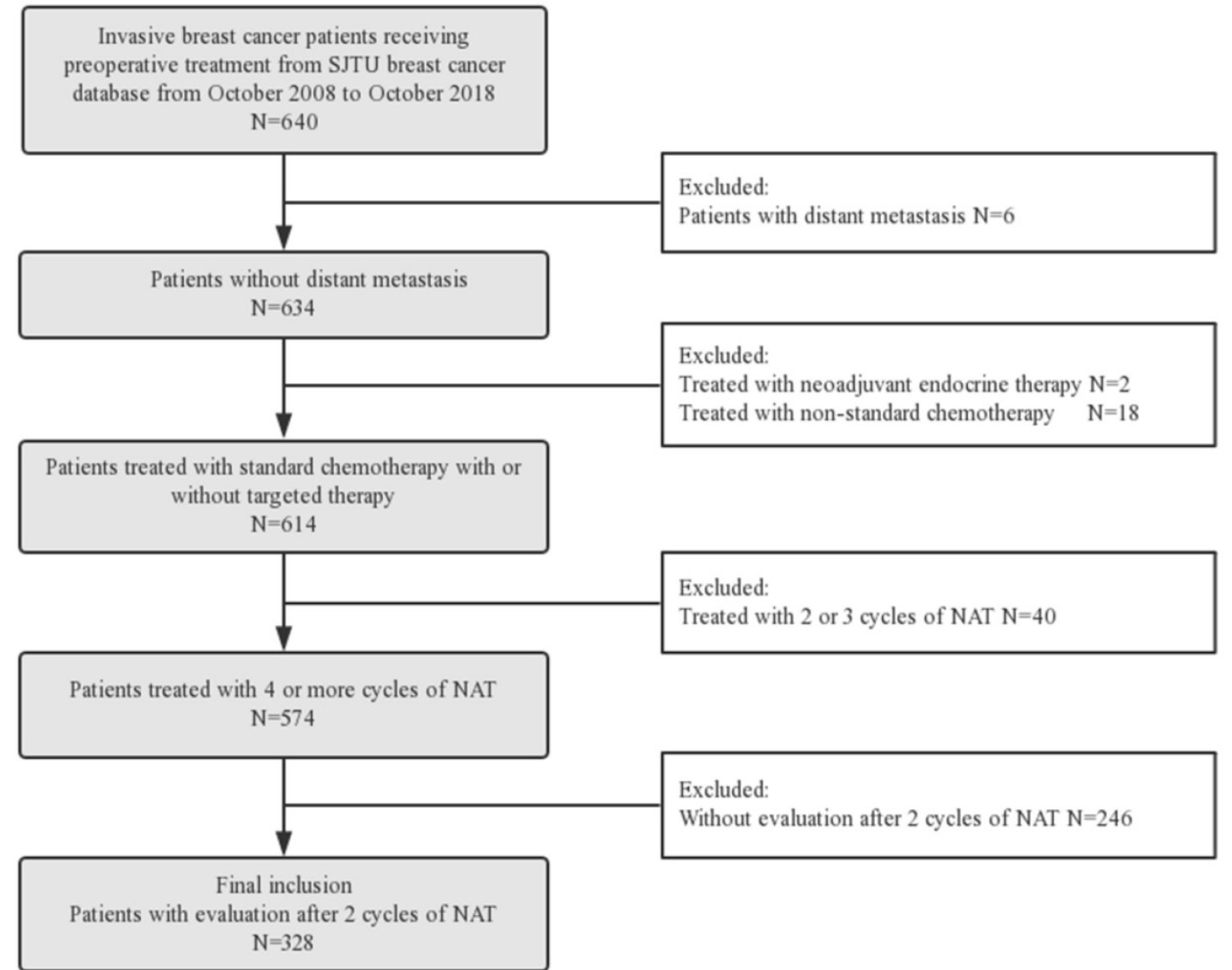

Figure 1. Patient Flow Diagram of the study. Abbreviation: NAT: neoadjuvant treatment.

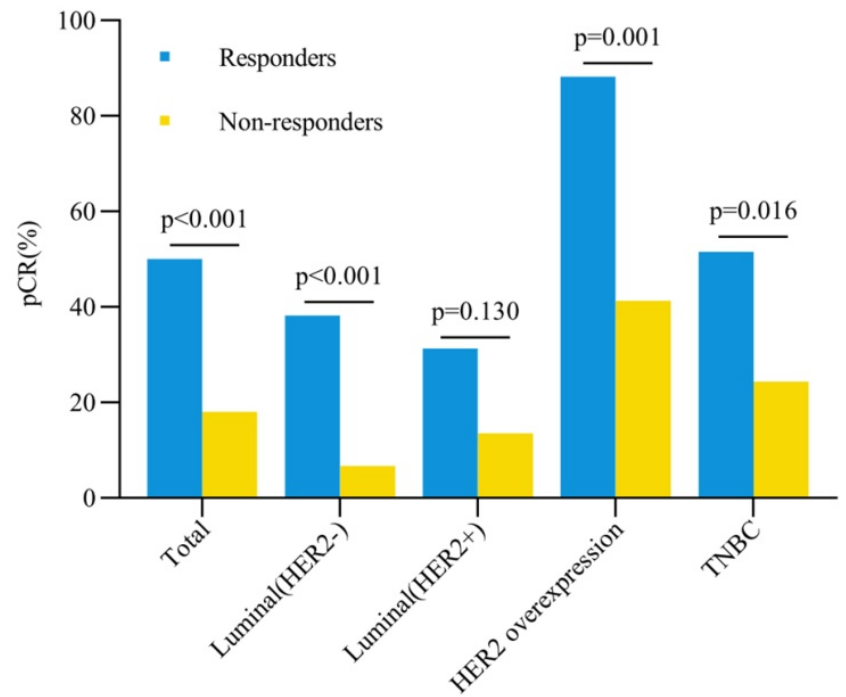

Figure 2. Breast pCR rate and early clinical response. Abbreviation: $p C R$ : pathological complete response, HER2: human epidermal growth factor receptor-2, TNBC: triple negative breast cancer.

\section{Clinical early response and the $\mathrm{PCR}$ rate}

The total pCR rate in breast was $50 \%$ for responders and $18 \%$ for non-responders $(P<0.001)$ (Figure 2). Univariate analysis showed that clinical early response, ER expression, PR expression, HER-2 status and targeted therapy were correlated with $\mathrm{pCR}$ rates after NAT (all $P<0.05$, Table 3). Logistic regression multivariate analysis revealed that clinical early response was an independent predictive factor for $\mathrm{pCR}$ in breast $(\mathrm{OR}=4.87,95 \% \mathrm{CI}=2.72-8.72, \mathrm{P}<$ 0.001 ) (Table 4). In addition, other factors were also associated with pCR in breast significantly, including ER status $(\mathrm{OR}=2.20,95 \% \mathrm{CI}=1.12-4.34, P=0.022)$, PR status $(\mathrm{OR}=2.68,95 \% \mathrm{CI}=1.13-6.33, P=0.025)$ and targeted therapy $(\mathrm{OR}=2.41,95 \% \mathrm{CI}=1.31-4.44, P=$ 0.005) (Table 4).

The $\mathrm{pCR}$ rates in breast for different subtypes were also further analyzed (Figure 2). Except the Luminal (HER2+) tumors, all three other subtypes showed higher $\mathrm{pCR}$ rates in responders than nonresponders. In patients with HER2 overexpression tumors, the pCR rate was as high as $88.2 \%$ for responders, which was significantly higher than $41.3 \%$ for non-responders $(\mathrm{OR}=10.66,95 \% \mathrm{CI}=$ 2.18-52.15, $P=0.001)$. In triple negative breast cancer patients, responders also had a significantly higher pCR rate than non-responders $(51.5 \%$ vs $24.4 \%$, OR = $3.29,95 \% \mathrm{CI}=1.23-8.84, P=0.016)$. Among patients with Luminal (HER2-) tumors, the pCR rate was $38.2 \%$ for responders and $6.7 \%$ for non-responders $(\mathrm{OR}=8.58,95 \% \mathrm{CI}=3.05-24.10, P<0.001)$. However, no significant difference was observed for $\mathrm{pCR}$ 
between responders and non-responders in Luminal (HER2+) patients $(31.3 \%$ vs $13.5 \%, \mathrm{OR}=2.90,95 \% \mathrm{CI}=$ $0.71-12.00, P=0.130)$. Besides, compared with other subtypes, patients with HER2 overexpression achieved the highest $\mathrm{pCR}$ rate both in responders $(P=$ 0.003 , Figure $3 \mathrm{~A})$ and non-responders $(P<0.001$, Figure 3B).

Table 3. Clinicopathological factors and $P C R$ in Breast

\begin{tabular}{|c|c|c|c|}
\hline Characteristics & pCR (No.) & Non-pCR (No.) & $P$-value \\
\hline Age & & & 0.890 \\
\hline$<50$ & 43 & 114 & \\
\hline$\geq 50$ & 48 & 123 & \\
\hline Pathology & & & 0.879 \\
\hline IDC & 75 & 197 & \\
\hline Others & 16 & 40 & \\
\hline Clinical $\mathrm{T}$ stage & & & 0.062 \\
\hline T0-2 & 79 & 184 & \\
\hline T3-4 & 12 & 53 & \\
\hline Clinical $\mathbf{N}$ stage & & & 0.653 \\
\hline N0-1 & 60 & 160 & \\
\hline N2-3 & 30 & 71 & \\
\hline ER status & & & $<0.001$ \\
\hline positive & 30 & 161 & \\
\hline negative & 61 & 76 & \\
\hline PR status & & & $<0.001$ \\
\hline positive & 11 & 111 & \\
\hline negative & 80 & 126 & \\
\hline HER2 status & & & 0.002 \\
\hline positive & 44 & 72 & \\
\hline negative & 47 & 165 & \\
\hline Regimen & & & 0.306 \\
\hline $\mathrm{A}$ or $\mathrm{T}$ & 24 & 50 & \\
\hline $\mathrm{A}$ and $\mathrm{T}$ & 67 & 187 & \\
\hline Target therapy & & & $<0.001$ \\
\hline Yes & 37 & 49 & \\
\hline No & 54 & 188 & \\
\hline Neoadjuvant Cycles & & & 0.751 \\
\hline 4 & 20 & 56 & \\
\hline$>4$ & 71 & 181 & \\
\hline Clinical early response & & & $<0.001$ \\
\hline Responders & 50 & 50 & \\
\hline Non-responders & 41 & 187 & \\
\hline
\end{tabular}

\section{Clinical early response and disease survival}

The median follow-up time was 43 months. The estimated 5-year DFS was $75.6 \%$ for responders and $66.4 \%$ for non-responders $(P=0.221)$ (Figure $4 \mathrm{~A})$. There was also no significant difference for the estimated 5-year OS between responders and nonresponders $(81.4 \%$ vs $84.2 \%, P=0.819)$ (Figure $4 \mathrm{~B})$. In terms of subgroup analysis according to pathological response, no significant differences were observed for the 5-year DFS or OS between responders and non-responders neither in the patients with $\mathrm{pCR}$ nor in patients with residual tumors (Figure 4C\&D, Table S1).

In responders, the estimated 5-year DFS was significantly higher for patients with $\mathrm{pCR}$ than patients not achieving pCR $(89.6 \%$ vs $60.9 \%, P=$ 0.0008) (Figure 3C, Table S2). Patients achieving pCR also had a significantly higher estimated 5-year OS than patients with residual tumors $(93.3 \%$ vs $74.0 \%, P$ $=0.010$ ) (Figure $4 \mathrm{D}$, Table S2). In non-responders, the estimated 5-year DFS was $81.2 \%$ for patients with pCR compared with $62.5 \%$ for patients with residual tumor $(P=0.066)$ and the estimated 5 -year OS was $83.7 \%$ for patients with $\mathrm{pCR}$ and $84.2 \%$ for patients without pCR $(P=0.405)$ (Figure $4 \mathrm{C} \& D$, Table S2).
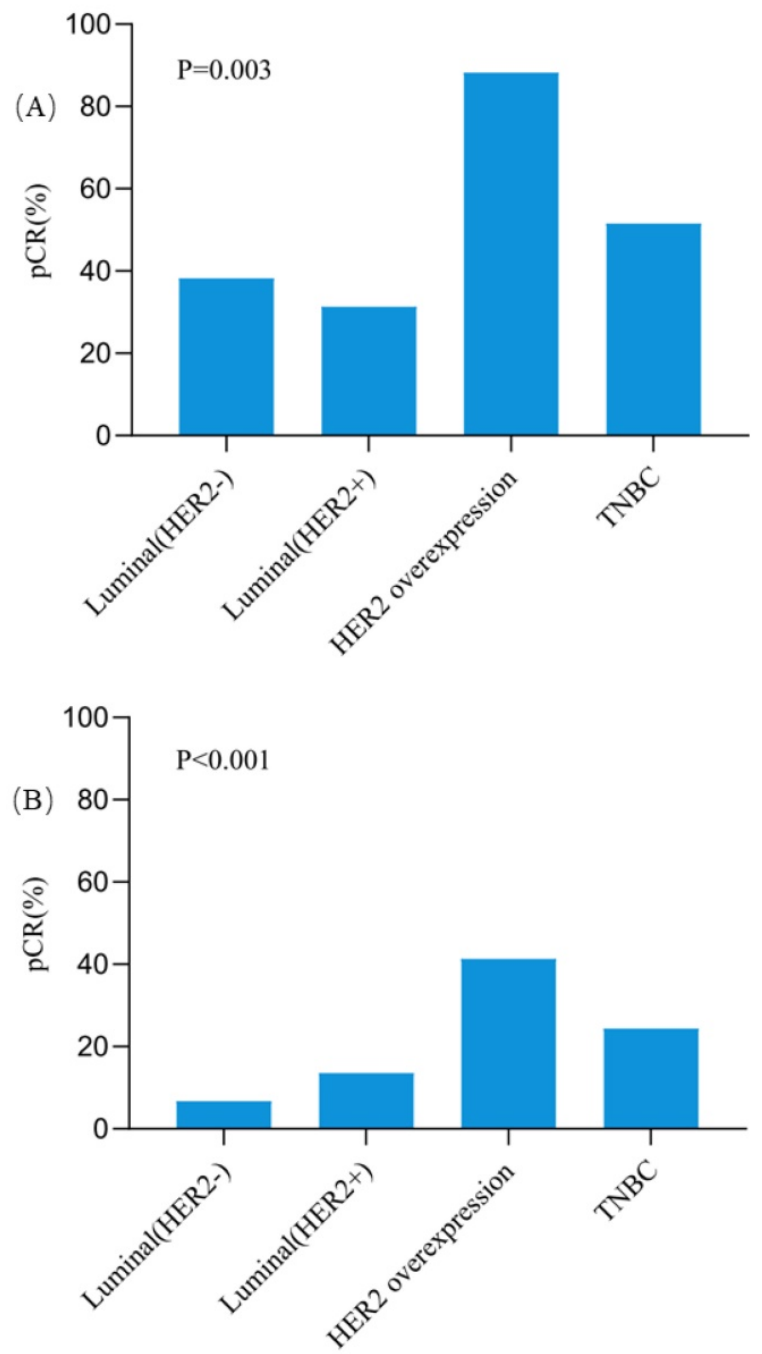

Figure 3. The $p C R$ rates in Breast among different molecular subtypes in clinically early responders (A) and non-responders (B). Abbreviation: $P C R$ : pathological complete response, HER2: human epidermal growth factor receptor-2, TNBC: triple negative breast cancer.

\section{Discussion}

Clinical early response to NAT was an excellent predictor for $\mathrm{pCR}$ in breast. Our current study showed that early response was significantly associated with a higher $\mathrm{pCR}$ rate in breast cancer patients receiving NAT, especially for patients with HER2 overexpression subtype. 

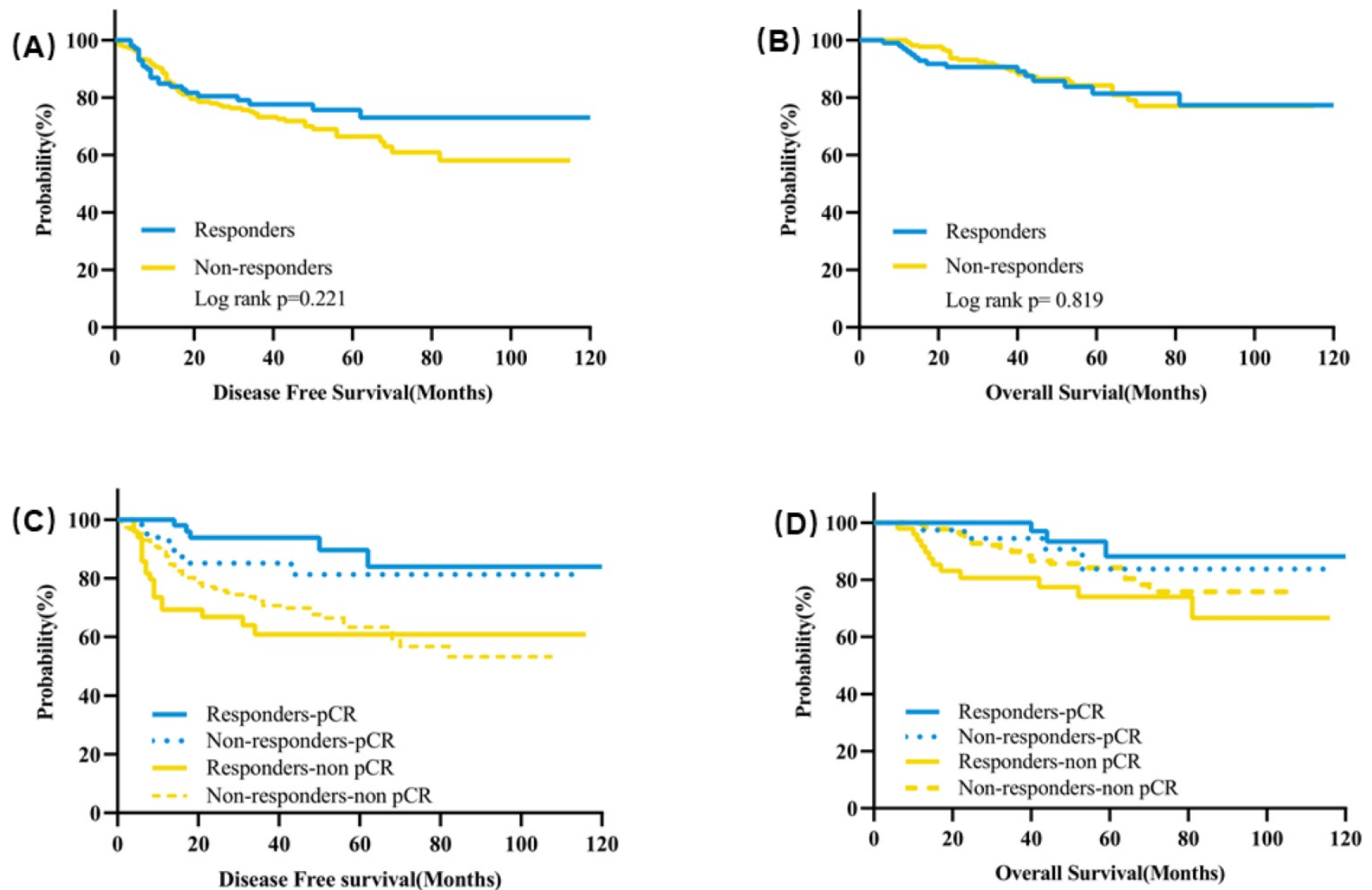

Figure 4. Kaplan-Meier curves of survival for clinical responders and non-responders after two cycles of NAT. (A) DFS according to clinical early response; (B) OS according to clinical early response; (C) DFS according to clinical early response and pathological response after NAT; (D) OS according to clinical early response and pathological response after NAT. Abbreviation: DFS: disease free survival, OS: overall survival, NAT: neoadjuvant treatment.

Table 4. Multivariate analysis of factors associated with $\mathrm{pCR}$ in Breast

\begin{tabular}{|c|c|c|c|}
\hline Characteristics & OR & $95 \% \mathrm{CI}$ & $p$-value \\
\hline \multicolumn{4}{|l|}{ ER status } \\
\hline positive & 1 & & \\
\hline negative & 2.20 & $1.12-4.34$ & 0.022 \\
\hline \multicolumn{4}{|l|}{ PR status } \\
\hline positive & 1 & & \\
\hline negative & 2.68 & $1.13-6.33$ & 0.025 \\
\hline \multicolumn{4}{|l|}{ HER2 status } \\
\hline negative & 1 & & \\
\hline positive & 1.14 & $0.48-2.71$ & 0.768 \\
\hline \multicolumn{4}{|l|}{ Target therapy } \\
\hline No & 1 & & \\
\hline Yes & 2.41 & $1.31-4.44$ & 0.005 \\
\hline \multicolumn{4}{|c|}{ Clinical early response } \\
\hline Non-responders & 1 & & \\
\hline Responders & 4.87 & $2.72-8.72$ & $<0.001$ \\
\hline
\end{tabular}

Neoadjuvant therapy is the preferred treatment of locally advanced breast cancer for the purpose of creating surgery opportunities [12]. An increasing number of operable breast cancer patients also receive NAT, which provides us an opportunity to observe the tumor shrink and response to neoadjuvant regimens, especially in the clinical trial for new drug development [1]. In the NeoSphere trial, the addition of pertuzumab to trastuzumab and docetaxel resulted in significantly higher $\mathrm{pCR}$ rates [13], accelerating the approval of pertuzumab in the neoadjuvant treatment of HER2 positive breast cancer by the Food and Drug Administration (FDA) [14]. Although the overall survival benefit of NAT remained controversial and even a higher local recurrence rate after breast conserving surgery was found in patients receiving NAT than those treated in the adjuvant setting [4], patients achieving $\mathrm{pCR}$ after NAT had better DFS and OS than patients with residual tumors $[5,6]$. Our study also confirmed that patients with pCR after NAT had significantly better DFS and OS than patients not achieving $\mathrm{pCR}$ in the responder group.

To date, three main methods were reported for early response assessment in neoadjuvant treatment of breast cancer: ${ }^{18} \mathrm{~F}-\mathrm{FDG}$ PET/CT, dynamic contrast enhanced MRI (DCE-MRI), and ultrasound $[9,15,16]$. Meta-analysis showed that ${ }^{18} \mathrm{~F}-\mathrm{FDG}$ PET/CT had a moderate accuracy for the early prediction for $\mathrm{PCR}$ (sensitivity $85 \%$, specificity $79 \%$ and diagnostic odds ratio 21.8), especially in HER2 positive and triple negative breast cancer $[16,17]$. DCE-MRI was also an 
effective method for early monitoring the efficacy during NAT (sensitivity $87 \%$, specificity $82 \%$ and diagnostic odds ratio 30.3) [18]. However, both ${ }^{18}$ F-FDG PET/CT and DCE-MRI require sophisticated imaging, additional radiation dose for patients, and less accessible compared with ultrasound. A systemic search in PubMed was performed, nine studies on the early prediction for pathological response by ultrasound in breast cancer are summarized in Table S4 [9, 19-26]. The diagnostic accuracy for pathological response with different parameters assessed by ultrasound during NAT was performed in eight studies and showed moderate results with different sensitivity and specificity. Among these studies, only two had estimated pCR rate different between early responders and non-responders, which were also not analyzed and compared among different subtypes [9, 19].

The pCR rates varied among different molecular subtypes. In a retrospective study with 13,939 breast cancer patients, patients with the Luminal A subtype had the lowest $\mathrm{pCR}$ rate $(0.3 \%)$ and the highest $\mathrm{pCR}$ rate was observed in HER-2 positive patients (38.7\%) [27]. Most of previous studies supported that patients with HER-2 positive breast cancer who received chemotherapy combination with targeted therapy achieved the highest $\mathrm{pCR}$ rate, especially those treated with dual targeted therapy $[8,27,28]$. The total clinical response rate was $30.5 \%$ in our study and responders had a significant higher $\mathrm{pCR}$ rate than non-responders (50\% vs $18 \%$ ). To our knowledge, no previous studies had ever focused on the predictive value of clinical early response for $\mathrm{pCR}$ in different molecular subtypes. In our study, its predictive value was largely independent of the molecular subtypes as except for Luminal (HER2+) group, responders had significantly higher $\mathrm{pCR}$ rates than non-responders in other three subgroups. The highest pCR rate was 54\% in HER2 overexpression patients and responders achieved a pCR rate of up to $88.2 \%$, which was analogous to patients receiving dual HER-2 blockade in combination with chemotherapy reported previously [13, 29-32]. The unexpected results indicated that patients with HER2 overexpression in breast cancer and have excellent early clinical response to NAT with chemotherapy and trastuzumab, do not need escalation of dual antiHER2 therapy. However, we also found that the Luminal (HER2+) subset was not benefit from clinical early response. Previous trials had showed that the pCR rates were higher in HER2+/HR- patients than HER2+/HR+ patients [32, 33]. Von Minckwitz and colleges also demonstrated that $\mathrm{pCR}$ was a surrogate end point for patients with triple negative, Luminal B/HER2-, and HER2 positive breast cancer but not for patients with Luminal B/HER2+ disease [34]. Therefore, the Luminal (HER2+) breast cancer was a special subtype that needed further clinical trials exploring novel systemic treatments. We also analyzed the pCR in breast and node (ypT0/isN0) and found the similar results in the whole population and different molecular subsets (Figure S1). Neoadjuvant regimens and treatment cycles may also influence treatment response [5, 35]. However, there were no differences for $\mathrm{pCR}$ rates between patients receiving different chemotherapy regimens or different NAT cycles in our study. While patients treated with trastuzumab had significantly higher pCR rates than those without trastuzumab treatment (Tables 3 and 4 ).

A previous study had also explored the predictive value of clinical response after two cycles of chemotherapy for $\mathrm{pCR}(P=0.003)$, in which responders included patients who had a $50 \%$ or greater reduction in tumor measurements [9]. In our study, however, responders were defined as patients with at least a $30 \%$ decrease of tumor size. The predictive value of different cut-off points of tumor size reduction (TSR) after two cycles of NAT had also been explored (Figure S2). Patients with TSR $\geq 20 \%$ had a higher pCR rate than patients with TSR $<20 \%$ (Breast: $43.1 \%$ vs $15.8 \%, P<0.001$; Breast and Node: $27.8 \%$ vs $10.9 \%, P<0.001)$. If responders were defined as patients with TSR $\geq 10 \%$, responders still had a significant higher $\mathrm{pCR}$ rate than non-responders (Breast: $37.6 \%$ vs $14.4 \%, P<0.001$; Breast and Node: $24.9 \%$ vs $9.4 \%, P<0.001)$. More importantly, the pCR rate deceased with the lower of the cut-off point of TSR and 30\% was an appropriate cut-off point for TSR in our study.

As clinical early response to NAT is correlated with $\mathrm{pCR}$, we analyzed factors associated with clinical early response and found that PR was an independent predictive factor. Patients with PR negative tumors had a 2.39 fold possibility to achieve early response than patients with PR positive disease. The prognostic value of clinical early response was also explored in our study and clinical early response could not translate into survival benefits. No significant differences were observed for DFS and OS between responders and non-responders irrespective of pathological response status. Meanwhile, in clinical responders, patients with $\mathrm{pCR}$ had significantly better DFS and OS than patients with residual tumors. Regarding non-responders, patients achieving pCR had a numerically higher DFS than patients with non$\mathrm{pCR}$, although there was no significant difference. Hence, pathological response can provide additional prognostic value for survival beyond clinical early response and clinical responders achieving $\mathrm{pCR}$ after 
NAT gained the greatest survival benefit. Moreover, what is the interference for non-responders? In the GeparTrio trial, after two cycles of TAC (docetaxel, doxorubicin and cyclophosphamide), early nonresponders were randomly assigned to an additional 4 cycles of TAC or to 4 cycles of NX (vinorelbine and capecitabine), the clinical response and $\mathrm{pCR}$ rates were similar between two groups[36]. However, DFS was higher in patients receiving TAC-NX than 6 cycles of TAC, which was mainly reflected in HR positive patients [37]. This implied that nonresponders after two cycles of NAT could receive an alternative regimen for better outcome.

There were some limitations of the study. Firstly, it was a retrospective study and neoadjuvant treatment regimens and cycles were diverse across patients. Secondly, as a single center analysis, patient numbers included in our study was not so large, especially for subgroup analysis. Thirdly, we could not validate our findings and conclusions with an independent cohort.

\section{Conclusions}

In conclusion, clinical responders after two cycles of NAT had a significantly higher pCR rate than non-responders. The predictive value of clinical early response was mainly observed in HER2 overexpression, triple negative, and Luminal (HER2-) breast cancer patients. Particularly, responders with HER2 overexpression subtype treated with trastuzumab had the highest $\mathrm{pCR}$ rate, which was analogous to those receiving dual HER2 blockade in previous literatures. Clinical early response after NAT may serve as an excellent marker for clinical decision making, deserving further clinical validation.

\section{Supplementary Material}

Supplementary figures and tables.

http://www.jcancer.org/v11p6916s1.pdf

\section{Acknowledgments}

The authors gratefully thank all the patients participating in this study and the staff at our center for their work and support of this study.

\section{Author contributions}

KWS and XSC developed the main concept and played an important role in the study design. $\mathrm{JH}$, JYW, OH, JRH, LZ, WGC and YFL contributed to the patients' data collection and follow-up. JH and XSC performed the data analysis and interpretation. $\mathrm{JH}$ drafted the manuscript. XSC and KWS contributed to editing and critical revision of the manuscript. All authors reviewed and approved the final draft.

\section{Funding}

The authors appreciated the financial support from the National Natural Science Foundation of China (Grant Number: 81772797), Shanghai Municipal Education Commission - Gaofeng Clinical Medicine Grant Support (20172007); Ruijin Hospital, Shanghai Jiao Tong University School of Medicine "Guangci Excellent Youth Training Program" (GCQN-2017-A18 and GCQN-2018-B11). All these financial sponsors had no role in the study design, data collection, analysis or interpretation.

\section{Ethical approval}

The protocol was reviewed and approved by the independent ethical committee/institutional review board of Shanghai Ruijin Hospital affiliated with Shanghai Jiao Tong University School of Medicine. All procedures performed in studies involving human participants were in accordance with the ethical standards of the committee and with the 1964 Helsinki declaration and its later amendments or comparable ethical standards.

\section{Competing Interests}

The authors have declared that no competing interest exists.

\section{References}

1. Vaidya JS, Massarut S, Vaidya HJ, Alexander EC, Richards T, Caris JA, et al. Rethinking neoadjuvant chemotherapy for breast cancer. BMJ. 2018; 360: j5913.

2. Untch M, Konecny GE, Paepke S, von Minckwitz G. Current and future role of neoadjuvant therapy for breast cancer. Breast. 2014; 23: 526-37.

3. De Mattos-Arruda L, Shen R, Reis-Filho JS, Cortes J. Translating neoadjuvant therapy into survival benefits: one size does not fit all. Nat Rev Clin Oncol. 2016; 13: 566-79.

4. Asselain B, Barlow W, Bartlett J, Bergh J, Bergsten-Nordström E, Bliss J, et al. Long-term outcomes for neoadjuvant versus adjuvant chemotherapy in early breast cancer: meta-analysis of individual patient data from ten randomised trials. The Lancet Oncology. 2018; 19: 27-39.

5. Rastogi P, Anderson SJ, Bear HD, Geyer CE, Kahlenberg MS, Robidoux A, et al. Preoperative chemotherapy: updates of National Surgical Adjuvant Breast and Bowel Project Protocols B-18 and B-27. J Clin Oncol. 2008; 26: 778-85.

6. Wang-Lopez Q, Chalabi N, Abrial C, Radosevic-Robin N, Durando X, Mouret-Reynier M-A, et al. Can pathologic complete response (pCR) be used as a surrogate marker of survival after neoadjuvant therapy for breast cancer? Critical Reviews in Oncology/Hematology. 2015; 95: 88-104.

7. Li XB, Krishnamurti U, Bhattarai S, Klimov S, Reid MD, O'Regan R, et al. Biomarkers Predicting Pathologic Complete Response to Neoadjuvant Chemotherapy in Breast Cancer. Am J Clin Pathol. 2016; 145: 871-8.

8. Gentile LF, Plitas G, Zabor EC, Stempel M, Morrow M, Barrio AV. Tumor Biology Predicts Pathologic Complete Response to Neoadjuvant Chemotherapy in Patients Presenting with Locally Advanced Breast Cancer. Ann Surg Oncol. 2017; 24: 3896-902.

9. Beresford MJ, Stott D, Makris A. Assessment of clinical response after two cycles of primary chemotherapy in breast cancer. Breast Cancer Res Treat. 2008; 109: 337-42.

10. Eisenhauer EA, Therasse P, Bogaerts J, Schwartz LH, Sargent D, Ford R, et al. New response evaluation criteria in solid tumours: revised RECIST guideline (version 1.1). Eur J Cancer. 2009; 45: 228-47.

11. Cortazar P, Geyer CE, Jr. Pathological complete response in neoadjuvant treatment of breast cancer. Ann Surg Oncol. 2015; 22: 1441-6.

12. Wang M, Hou L, Chen M, Zhou Y, Liang Y, Wang S, et al. Neoadjuvant Chemotherapy Creates Surgery Opportunities For Inoperable Locally Advanced Breast Cancer. Sci Rep. 2017; 7: 44673.

13. Gianni L, Pienkowski T, Im Y-H, Roman L, Tseng L-M, Liu M-C, et al. Efficacy and safety of neoadjuvant pertuzumab and trastuzumab in women with locally advanced, inflammatory, or early HER2-positive breast cancer (NeoSphere): a randomised multicentre, open-label, phase 2 trial. The Lancet Oncology. 2012; 13: 25-32. 
14. Haddad TC, Goetz MP. Landscape of neoadjuvant therapy for breast cancer. Ann Surg Oncol. 2015; 22: 1408-15.

15. Prevos R, Smidt ML, Tjan-Heijnen VC, van Goethem M, Beets-Tan RG, Wildberger JE, et al. Pre-treatment differences and early response monitoring of neoadjuvant chemotherapy in breast cancer patients using magnetic resonance imaging: a systematic review. Eur Radiol. 2012; 22: 2607-16.

16. Tian F, Shen G, Deng Y, Diao W, Jia Z. The accuracy of (18)F-FDG PET/CT in predicting the pathological response to neoadjuvant chemotherapy in patients with breast cancer: a meta-analysis and systematic review. Eur Radiol. 2017; 27: 4786-96.

17. Groheux D, Mankoff D, Espie M, Hindie E. (1)(8)F-FDG PET/CT in the early prediction of pathological response in aggressive subtypes of breast cancer: review of the literature and recommendations for use in clinical trials. Eur J Nucl Med Mol Imaging. 2016; 43: 983-93.

18. Cheng $Q$, Huang J, Liang J, Ma M, Ye K, Shi C, et al. The Diagnostic Performance of DCE-MRI in Evaluating the Pathological Response to Neoadjuvant Chemotherapy in Breast Cancer: A Meta-Analysis. Front Oncol. 2020; 10: 93 .

19. Di Cosimo S, Campbell C, Azim HA, Jr., Galli G, Bregni G, Curigliano G, et al. The use of breast imaging for predicting response to neoadjuvant lapatinib, trastuzumab and their combination in HER2-positive breast cancer: Results from Neo-ALTTO. Eur J Cancer. 2018; 89: 42-8.

20. Falou O, Sadeghi-Naini A, Prematilake S, Sofroni E, Papanicolau N, Iradji S, et al. Evaluation of neoadjuvant chemotherapy response in women with locally advanced breast cancer using ultrasound elastography. Transl Oncol. 2013; 6: $17-24$.

21. Fernandes J, Sannachi L, Tran WT, Koven A, Watkins E, Hadizad F, et al. Monitoring Breast Cancer Response to Neoadjuvant Chemotherapy Using Ultrasound Strain Elastography. Transl Oncol. 2019; 12: 1177-84.

22. Jing H, Cheng W, Li ZY, Ying L, Wang QC, Wu T, et al. Early Evaluation of Relative Changes in Tumor Stiffness by Shear Wave Elastography Predicts the Response to Neoadjuvant Chemotherapy in Patients With Breast Cancer. J Ultrasound Med. 2016; 35: 1619-27.

23. Lee YJ, Kim SH, Kang BJ, Kim YJ. Contrast-Enhanced Ultrasound for Early Prediction of Response of Breast Cancer to Neoadjuvant Chemotherapy. Ultraschall Med. 2019; 40: 194-204.

24. Marinovich ML, Houssami N, Macaskill P, von Minckwitz G, Blohmer JU, Irwig L. Accuracy of ultrasound for predicting pathologic response during neoadjuvant therapy for breast cancer. Int J Cancer. 2015; 136: 2730-7.

25. Sannachi L, Tadayyon H, Sadeghi-Naini A, Tran W, Gandhi S, Wright F, et al. Non-invasive evaluation of breast cancer response to chemotherapy using quantitative ultrasonic backscatter parameters. Med Image Anal. 2015; 20: 224-36.

26. Wang X, Huo L, He Y, Fan Z, Wang T, Xie Y, et al. Early prediction of pathological outcomes to neoadjuvant chemotherapy in breast cancer patients using automated breast ultrasound. Chinese Journal of Cancer Research. 2016; 28: 478-85.

27. Haque W, Verma V, Hatch S, Suzanne Klimberg V, Brian Butler E, Teh BS. Response rates and pathologic complete response by breast cancer molecular subtype following neoadjuvant chemotherapy. Breast Cancer Res Treat. 2018; 170: 559-67.

28. van Uden DJP, van Maaren MC, Bult P, Strobbe LJA, van der Hoeven JJM, Blanken-Peeters C, et al. Pathologic complete response and overall survival in breast cancer subtypes in stage III inflammatory breast cancer. Breast Cancer Res Treat. 2019; 176: 217-26.

29. de Azambuja E, Holmes AP, Piccart-Gebhart M, Holmes E, Di Cosimo S, Swaby RF, et al. Lapatinib with trastuzumab for HER2-positive early breast cancer (NeoALTTO): survival outcomes of a randomised, open-label, multicentre, phase 3 trial and their association with pathological complete response. The Lancet Oncology. 2014; 15: 1137-46.

30. Carey LA, Berry DA, Cirrincione CT, Barry WT, Pitcher BN, Harris LN, et al. Molecular Heterogeneity and Response to Neoadjuvant Human Epidermal Growth Factor Receptor 2 Targeting in CALGB 40601, a Randomized Phase III Trial of Paclitaxel Plus Trastuzumab With or Without Lapatinib. Journal of Clinical Oncology. 2016; 34: 542-9.

31. Swain SM, Ewer MS, Viale G, Delaloge S, Ferrero JM, Verrill M, et al. Pertuzumab, trastuzumab, and standard anthracycline- and taxane-based chemotherapy for the neoadjuvant treatment of patients with HER2-positive localized breast cancer (BERENICE): a phase II, open-label, multicenter, multinational cardiac safety study. Ann Oncol. 2018; 29: 646-53.

32. Harbeck N, Gluz O. Neoadjuvant therapy for triple negative and HER2-positive early breast cancer. Breast. 2017; 34 Suppl 1: S99-S103.

33. Schneeweiss A, Chia S, Hickish T, Harvey V, Eniu A, Hegg R, et al. Pertuzumab plus trastuzumab in combination with standard neoadjuvant anthracycline-containing and anthracycline-free chemotherapy regimens in patients with HER2-positive early breast cancer: a randomized phase II cardiac safety study (TRYPHAENA). Ann Oncol. 2013; 24: 2278-84.

34. von Minckwitz G, Untch M, Blohmer JU, Costa SD, Eidtmann H, Fasching PA, et al. Definition and impact of pathologic complete response on prognosis after neoadjuvant chemotherapy in various intrinsic breast cancer subtypes. J Clin Oncol. 2012; 30: 1796-804.

35. Han S, Kim J, Lee J, Chang E, Gwak G, Cho H, et al. Comparison of 6 cycles versus 4 cycles of neoadjuvant epirubicin plus docetaxel chemotherapy in stages II and III breast cancer. Eur J Surg Oncol. 2009; 35: 583-7.
36. von Minckwitz G, Kummel S, Vogel P, Hanusch C, Eidtmann H, Hilfrich J, et al. Neoadjuvant vinorelbine-capecitabine versus docetaxel-doxorubicincyclophosphamide in early nonresponsive breast cancer: phase III randomized GeparTrio trial. J Natl Cancer Inst. 2008; 100: 542-51.

37. von Minckwitz G, Blohmer JU, Costa SD, Denkert C, Eidtmann H, Eiermann $\mathrm{W}$, et al. Response-guided neoadjuvant chemotherapy for breast cancer. J Clin Oncol. 2013; 31: 3623-30. 